\title{
Análisis de las interconsultas realizadas al Servicio de Dermatología del Hospital Central de Mendoza
}

\section{Analysis of the medical consultations performed at the Hospital Central de Mendoza's Dermatology Service}

Gabriela Anahí Hernández Barrios ${ }^{1}$, Rocío Celeste Muñiz Adaro ${ }^{2}$, Paula Melisa Benitez ${ }^{3}$, Laura Flores ${ }^{4}$ y Sofía Andrés

\section{RESUMEN}

Antecedentes: La dermatología se ha considerado clásicamente una especialidad limitada al ámbito ambulatorio. Sin embargo, los pacientes ingresados presentan con frecuencia afecciones cutáneas asociadas o no a enfermedades sistémicas.

Objetivo: Analizar las características de las interconsultas realizadas al Servicio de Dermatología del Hospital Central de Mendoza.

Diseño: Estudio observacional analítico de corte longitudinal. Materiales y métodos: Se incluyeron 425 interconsultas de pacientes hospitalizados y ambulatorios que requirieron una evaluación dermatológica urgente, desde el 1 de febrero hasta el 31 agosto de 2019.

Resultados: Se analizaron 266 (62,59\%) interconsultas de pacientes ambulatorios con requerimiento de evaluación dermatológica urgente, de ellas 228 (85,71\%) fueron derivados del Servicio de Guardia. Del total, 159 $(37,41 \%)$ fueron interconsultas de pacientes hospitalizados, de las cuales 74 (46,54\%) fueron solicitadas por Clínica Médica. Las dermatopatías in- fecciosas fueron las más frecuentes (38,82\%), seguidas de las inflamatorias $(37,65 \%)$. Las lesiones cutáneas asociadas a enfermedad sistémica fueron más frecuentes en los internados $(p=0,003)$. Del total de las interconsultas, $230(54,11 \%)$ contaban con un diagnóstico presuntivo y, de ellas, 127 $(55,21 \%)$ coincidieron con el diagnóstico definitivo realizado por el dermatólogo. Se comparó la coincidencia entre el diagnóstico presuntivo y el definitivo entre grupos de médicos con orientación clínica frente a quirúrgica y se halló coincidencia en el 30,95\% y el 23,40\% de los casos, en ese orden, sin observarse diferencias estadísticamente significativas $(p=0,28)$. Conclusión: La evaluación dermatológica facilita el diagnóstico y el manejo del paciente en el contexto hospitalario.

Palabras clave: interconsultas médicas, pacientes hospitalizados, pacientes ambulatorios, dermatología.

Dermatol. Argent. 2020, 26 (2): 58-62

\section{ABSTRACT}

Background: Dermatology has classically been considered a medical specialty confined to the ambulatory scope. Nevertheless, patients admitted frequently present skin conditions that can be associated or not to a systemic disease.

Objective: To analyze the medical consultation performed at the Hospital Central de Mendoza's Dermatology Service.

Design: Observational, analytical, longitudinal section study.

Materials and methods: In this study were included 425 medical consultations from hospitalized and ambulatory patients, that required urgent dermatological examination, from the 1st of February to the 31st of August, 2019.

Results: 266 (62.59\%) medical consultations from ambulatory patients requiring urgent dermatological examination were analyzed, and from them, 228 (85.71\%) were referred from the medical on-call service. 159 (37.41\%) consisted in hospitalized patients' consultations, from which 74 (46.54\%) were requested by the Internal Medicine Service. The infectious skin diseases were the most frequent (38.82\%), followed by the inflammatory skin diseases (37.65\%). Skin lesions associated with systemic diseases were more frequent in the inpatients $(p=0.003)$. From the total of medical consultations, $230(54.11 \%)$ had a presumptive diagnosis, where 127 (55.21\%) of the cases matched the definitive diagnosis. Although clinicians made more accurate presumptive diagnoses than surgeons ( $30.95 \%$ vs. $23.40 \%$, respectively), there was no statistically significant difference between them $(p=0.28)$.

Conclusion: Dermatological examination facilitates the diagnosis and patient's management in the hospital context.

Key words: medical consultations, hospitalized patients, outpatients, dermatology.

Dermatol. Argent. 2020, 26 (2): 58-62 
1 Residente de Cuarto Año

2 Jefa de Residentes

${ }^{3}$ Residente de Tercer Año

${ }^{4}$ Residente de Cuarto Año, Servicio de Clínica Médica

${ }^{5}$ Médica de Planta

Servicio de Dermatología, Hospital Central, Mendoza, Provincia de Mendoza, Argentina

\section{INTRODUCCIÓN}

La dermatología se ha considerado siempre una especialidad limitada al ámbito ambulatorio. Sin embargo, pese a que los motivos de ingreso exclusivamente dermatológicos son escasos, las dermatosis más comunes y los signos cutáneos asociados a una enfermedad sistémica suelen estar presentes en los pacientes hospitalizados. Asimismo, es usual la existencia de intercurrencias dermatológicas en pacientes atendidos por otras patologías. Por lo tanto, el valor del dermatólogo como interconsultor dentro del entorno hospitalario es de vital importancia.

Nuestro equipo de trabajo se desarrolla en el Hospital Central de Mendoza, Argentina. Este representa la institución pública más grande de la provincia y funciona como centro de referencia de nivel terciario en la región de Cuyo de nuestro país. Cuenta con 347 camas para internación, en su mayoría correspondientes a los servicios de Medicina Interna y Cirugía General. Además, el departamento de Guardia se halla en constante actividad, con consultas que rondan entre $240 \mathrm{y}$ 270 por día.

Dada la gran cantidad de consultas que recibimos, realizamos este trabajo con el objetivo de analizar las características de las interconsultas efectuadas al Servicio de Dermatología. Si bien se han publicado numerosos artículos sobre el perfil de las interconsultas realizadas al médico dermatólogo en este ámbito, no encontramos estudios de este tipo en la Argentina.

\section{MATERIALES Y MÉTODOS}

\section{Población}

Se incluyeron todas las interconsultas (IC) de pacientes con lesiones dermatológicas, tanto hospitalizados como ambulatorios, provenientes de la guardia o de otros servicios recibidas desde el 1 de febrero hasta el 31 agosto de 2019. En cuanto a la edad, se incluyeron todos los individuos mayores de 15 años.

\section{Diseño del estudio}

Estudio observacional analítico de corte longitudinal.
Contacto del autor: Gabriela Anahí Hernández Barrios

E-mail: gabyhernandezba@gmail.com

Fecha de trabajo recibido: 5/10/2019

Fecha de trabajo aceptado: 2/5/2020

Conflicto de interés: las autoras declaran que no existe conflicto de interés.

\section{Análisis de variables}

Se evaluaron de manera prospectiva 425 solicitudes de interconsultas, respondidas dentro de las 24 horas de su emisión. En todas ellas las variables analizadas incluyeron datos demográficos, antecedentes patológicos, servicio interconsultor, diagnóstico presuntivo y diagnóstico definitivo. Las lesiones cutáneas de cada paciente evaluado se clasificaron en limitadas a la piel y asociadas a enfermedad sistémica. Además, se agruparon en entidades inflamatorias, infecciosas, neoplásicas, farmacodermias, colagenopatías y otras. Los diagnósticos provisionales formulados por el equipo solicitante se compararon con los diagnósticos dermatológicos finales. En la evaluación del diagnóstico presuntivo realizado por el médico efector y el definitivo por el dermatólogo, consideramos coincidente tanto cuando el diagnóstico fue igual como cuando se encontraba dentro del mismo grupo etiológico. Los exámenes complementarios, la necesidad de tratamiento tópico o sistémico y la derivación a otra especialidad fueron aspectos también estudiados. En los pacientes internados, además, se especificó el motivo de ingreso y el día de internación en el que se solicitó la interconsulta.

Para valorar si la formación del médico efector tenía influencia en el diagnóstico presuntivo dermatológico, se categorizaron en dos grupos: médicos con orientación clínica (pertenecientes a clínica médica, hematología, oncología, nefrología, endocrinología, infectología, inmunología, neumología, cardiología, unidad coronaria, unidad de cuidados intensivos, hepatología, hospital de un día, personal del hospital y guardia) y médicos con orientación quirúrgica (pertenecientes a cirugía general, traumatología, cirugía cardiovascular, cirugía vascular periférica, oftalmología, odontología y otorrinolaringología).

\section{Análisis estadístico}

Las variables continuas se expresaron como media ( \pm desviación estándar). Las variables categóricas se expresaron como frecuencias absolutas y relativas, y se compararon mediante la prueba de la chi al cuadrado. Las medidas de asociación se expresaron con su riesgo relativo (OR), con un intervalo de confianza del $95 \%$. 
Un valor de $p$ menor de 0,05 se consideró significativo. Los análisis estadísticos se realizaron con Epi Info 7.

La elaboración del presente trabajo contó con la aprobación del Comité de Ética e Investigación del Hospital Central de Mendoza.

\section{RESULTADOS}

Se examinaron 425 interconsultas, de las cuales 261 $(61,41 \%)$ fueron de varones y 164 (38,59\%), de mujeres. El promedio de edad fue de 40,37 años (DE: 15,67), con un rango 15 a 92 ańos.

En los pacientes internados, la mediana de días de internación en que se realizó la IC fue de 5 , con un rango intercuartílico de 2 y 10 . La media fue de un día. De estos 159 pacientes, 19 (11,94\%) ingresaron por afecciones cutáneas.

De las 425 IC, $266(62,59 \%)$ correspondieron a pacientes ambulatorios con requerimiento de evaluación dermatológica. De estos, 228 (85,71\%) fueron derivados de la guardia. En la Tabla 1 se presenta la distribución de interconsultas por servicio.

Las interconsultas de pacientes internados fueron 159 (37,41\%). El departamento que más IC realizó fue el de Clínica Médica con 74 (46,54\%) solicitudes, seguidas del de Neurocirugía con 12 (7,55\%). El resto de las interconsultas por servicio se describen en la Tabla 2.

En $27(16,98 \%)$ de los pacientes internados, la IC se efectuó dentro de las primeras 24 horas de hospitalización. En 19 (11,94\%) el motivo de internación fue una dermatosis.

Se clasificaron en grupos según su etiología en infecciosas $(165 ; 38,82 \%)$, inflamatorias $(160 ; 37,65 \%)$, farmacodermias $(20 ; 4,70 \%)$, neoplásicas (18; 4,24\%), colagenopatías $(6 ; 1,41 \%)$ y otras $(56 ; 13,18 \%)$.

Las 11 dermatopatías que resultaron más frecuentes fueron: eccema en 66 pacientes $(15,53 \%)$, sífilis en 20 (4,71\%), prurigo en 19 (4,47\%), dermatofitosis en 17 (4\%), impétigo en 14 (3,29\%), herpes zóster en 13 (3,06\%), herpes simple en $13(3,06 \%)$, escabiosis en 12 $(2,82 \%)$, psoriasis en $10(2,35 \%)$, urticaria en $7(1,65 \%)$ y celulitis en 7 pacientes $(1,65 \%)$.

Además, del total de interconsultas, 371 (87,29\%) diagnósticos efectuados correspondieron a patologías limitadas a la piel, de los cuales $242(65,23 \%)$ correspondieron a ambulatorios y 129 (34,77\%), a internados. Las restantes 54 (12,71\%) se asociaron a compromiso sistémico, correspondiendo $24(9,02 \%)$ a ambulatorios y $30(18,87 \%)$ a internados. Es decir, en los pacientes internados se realizaron más diagnósticos de lesiones cutáneas asociadas a enfermedad sistémica respecto de los ambulatorios $(\mathrm{OR}=2,34$; IC 95\%: $1,31$ a 4,$20 ; p=0,003)$.

\begin{tabular}{|l|c|c|}
\hline \multicolumn{1}{|c|}{ Servicios ambulatorios } & N & $\begin{array}{c}\text { Porcentaje } \\
\mathbf{1 0 0 \%}\end{array}$ \\
\hline Guardia & 228 & 85,70 \\
\hline Oftalmología & 9 & 3,37 \\
\hline Hematología & 9 & 3,37 \\
\hline Oncología & 4 & 1,50 \\
\hline Odontología & 2 & 0,75 \\
\hline Otorrinolaringología & 2 & 0,75 \\
\hline Cardiología & 1 & 0,38 \\
\hline Cirugía general & 1 & 0,38 \\
\hline Endocrinología & 1 & 0,38 \\
\hline Ginecología & 1 & 0,38 \\
\hline Hepatología & 1 & 0,38 \\
\hline Infectología & 1 & 0,38 \\
\hline Inmunología & 1 & 0,38 \\
\hline Nefrología & 1 & 0,38 \\
\hline Neumología & 1 & 0,38 \\
\hline Neurocirugía & 1 & 0,38 \\
\hline Urología & 1 & 0,38 \\
\hline Consultorio de personal hospitalario & 1 & 0,38 \\
\hline TABLA 1: Distribución de los pacientes ambulatorios. & \\
\hline & & \\
\hline
\end{tabular}

\begin{tabular}{|l|c|c|}
\hline \multicolumn{1}{|c|}{ Servicios de internación } & N & $\begin{array}{c}\text { Porcentaje } \\
\mathbf{1 0 0 \%}\end{array}$ \\
\hline Clínica Médica & 74 & 46,54 \\
\hline Neurocirugía & 12 & 7,55 \\
\hline Nefrología & 11 & 6,92 \\
\hline Hospital de un día & 11 & 6,92 \\
\hline Cardiología & 10 & 6,29 \\
\hline Hematología & 9 & 5,66 \\
\hline Unidad de terapia intensiva & 8 & 5,03 \\
\hline Cirugía general & 8 & 5,03 \\
\hline Unidad coronaria & 6 & 3,77 \\
\hline Ginecología & 5 & 3,14 \\
\hline Traumatología & 2 & 1,26 \\
\hline Recuperación cardiovascular & 1 & 0,63 \\
\hline Cirugía vascular periférica & 1 & 0,63 \\
\hline Urología & 1 & 0,63 \\
\hline TABLA 2: Distribución de los pacientes internados. & \\
\hline
\end{tabular}

En relación con el diagnóstico presuntivo efectuado por el médico no dermatólogo, no se especificó en 195 pacientes $(45,88 \%)$. De estos, 106 (54,35\%) no contaban con una presunción diagnóstica y en 89 (45,64\%) 
casos se incluyeron términos no diagnósticos como "lesión en la piel", "erupción”, "dermatitis”, "dermatosis" o simplemente una descripción de la lesión clínica por evaluar. De los $230(54,12 \%)$ pacientes que contaban con un diagnóstico presuntivo, 127 (55,21\%) coincidieron con el diagnóstico definitivo efectuado por el dermatólogo. Realizaron 378 (88,94\%) y $47(11,06 \%)$ IC, respectivamente. Se comparó la coincidencia entre el diagnóstico presuntivo y el definitivo y se halló coincidencia en 117 casos $(30,95 \%)$ en las especialidades clínicas y en $11(23,40 \%)$ en las especialidades quirúrgicas, sin observarse diferencia significativa entre ambos grupos $(p=0,28)$.

En referencia a la solicitud de exámenes complementarios, de las 266 IC ambulatorias, 71 (26,7\%) requirieron estudios complementarios y de los internados, $46(28,93 \%)$. De los pacientes ambulatorios, solo a $7(2,63 \%)$ se les realizó biopsia de piel y a 2 pacientes $(0,75 \%)$, cultivo microbiológico. De los pacientes internados, $15(9,43 \%)$ y $6(3,77 \%)$, respectivamente.

Se indicó tratamiento a $239(89,84 \%)$ de los pacientes ambulatorios, solo tópico a $89(37,24 \%)$, solo sistémico a $74(30,96 \%)$ y ambos a $76(31,80 \%)$. Asimismo, en 116 (72,95\%) pacientes internados se inició tratamiento, solo tópico en $67(57,76 \%)$, solo sistémico en 31 (26,72\%) y ambos en 18 (15,52\%). Se solicitó derivación a otra especialidad en 28 casos de total de IC evaluadas, 12 (4,51\%) en pacientes ambulatorios y $16(10,06 \%)$, en internados.

\section{COMENTARIOS}

En las IC examinadas se observó un predominio del sexo masculino con una relación varón: mujer de $1,6: 1$, al igual que en la literatura especializada ${ }^{1,2}$.

Respecto de los pacientes internados, la mayor cantidad de IC se solicitaron por parte del servicio de Clínica Médica, al igual que en otros estudios ${ }^{2-4}$. Cabe aclarar que es el servicio de internación que además tiene mayor número de camas disponibles. En segundo lugar, se encontró el servicio de Neurocirugía, lo cual no se condice con la bibliografía, pero destacamos que la mayoría de los pacientes evaluados en interconsulta de este servicio fueron por reacciones adversas a fármacos, lo cual sí se relaciona con la medicación habitualmente indicada en este tipo de enfermos. La gran cantidad de pacientes derivados de la guardia para evaluación urgente en este período refleja, por un lado, la necesidad de la interacción entre el médico solicitante y el especialista de piel para el manejo del paciente y, por otro lado, un importante número de consultas al servicio de emergencia por patologías cutáneas banales.
Las enfermedades cutáneas infecciosas fueron las más frecuentes, seguidas de las inflamatorias. Esto coincide con lo publicado en la literatura ${ }^{1,2,4-7}$. El diagnóstico de eccema fue el más frecuente, lo que podría asociarse a la alta prevalencia propia de esta dermatosis y también a la mayor exposición a antisépticos, sudoración por decúbito dorsal permanente, uso de pañales y vendajes oclusivos en el caso de los pacientes hospitalizados $^{1,8}$. Debe alertarnos que el diagnóstico de sífilis se encuentre en el segundo lugar del total de IC analizadas. Este hallazgo muestra una realidad preocupante que se relaciona con un importante salto en la escala de informes de casos de sífilis registrados a nivel nacional". A pesar de ser conocida en la medicina como "la gran simuladora" por su amplia variedad de presentaciones clínicas, el médico no dermatólogo no suele considerarla en sus diagnósticos diferenciales, excepto en sus formas clínicas más típicas. Además, y como era esperable encontrar, en los pacientes internados se realizaron más diagnósticos de lesiones cutáneas asociadas a compromiso sistémico respecto de los ambulatorios $(p=0,003)$.

A menudo, no se reconocen las enfermedades comunes de la piel o suelen confundirse con otros diagnósticos cuando las evalúan profesionales no dermatólogos. Falanga et ál. evidenciaron en su trabajo que el 78\% de los diagnósticos efectuados por médicos no especialistas de piel habían sido erróneos ${ }^{2,6,10,11}$. Esto mismo se repite en otros estudios realizados, con porcentajes similares a nuestros resultados ${ }^{5,12}$. Pese a tener la percepción de que los médicos con especialidades clínicas estaban más orientados hacia el diagnóstico definitivo que aquellos con especialidades quirúrgicas, la diferencia entre ambos grupos no fue estadísticamente significativa. A partir de ello, podemos inferir que este déficit probablemente obedezca al poco tiempo dedicado a la docencia de la dermatología en el período de formación universitaria del médico generalista, lo que favorece que, incluso, no se identifiquen afecciones dermatológicas comunes $5^{5,10,11,13}$.

Respecto de la solicitud de exámenes complementarios, no hallamos diferencia significativa entre los pacientes internados y los ambulatorios $(p=0,61)$. Este resultado puede deberse a que en el momento de la evaluación, el paciente internado ya cuenta con análisis de laboratorio realizados previamente ${ }^{14}$. El dermatólogo suele realizar un diagnóstico clínico sin necesidad de otras pruebas, a veces solicitadas por otros médi$\cos ^{12}$. En este trabajo, la mayoría de los diagnósticos se establecieron de forma clínica y se realizó la toma de biopsia cutánea solo en 22 pacientes, con predominio de los pacientes internados. 


\section{LIMITACIONES}

Se debieron unificar los diagnósticos presuntivos del médico no dermatólogo por ser muy variados y poco específicos en un gran número de casos. Se analizaron los principales diagnósticos definitivos de piel (uno por paciente), pero muchos casos contaban con más de un diagnóstico dermatológico. Otra limitación importante de este trabajo fue no consignar si se había iniciado tratamiento previo, lo que impidió que se analizara si hubo un cambio de conducta terapéutica, y si tuvo o no consecuencias en la evolución del paciente. En relación con esto último, tampoco se realizó un seguimiento longitudinal de los casos ni se detalló el número de pacientes que fueron internados después de la evaluación ambulatoria.

\section{CONCLUSIÓN}

En los últimos años, la actividad del dermatólogo ha experimentado una gran ampliación de su campo de trabajo. Sin embargo, sigue siendo una figura escasamente valorada en el ámbito hospitalario ${ }^{7}$.

\section{BIBLIOGRAFÍA}

1. Lorente-Lavirgen Al, Bernabeu-Wittel J, Pulpillo-Ruiz A, De la Torre-García JM, et ál. Interconsulta hospitalaria en Dermatología sobre una cohorte prospectiva en un hospital español de tercer nivel. Actas Dermosifiliogr 2013;104:148-155.

2. Storan E, McEvoy M, Wetter D, El-Azhary R, et ál. Experience of a year of adult hospital dermatology consultations. Int J Dermatol 2015;54:1150-1156.

3. Galimberti F, Guren L, Fernandez AP, Sood A. Dermatology consultations significantly contribute quality to care of hospitalized patients: a prospective study of dermatology inpatient consults at a tertiary care center. Int $J$ Dermatol 2016;76:169-173.

4. Cavero-Guardamino JA. Características demográficas y clínicas de los pacientes evaluados en interconsulta dermatológica en un hospital nacional de Lima-Perú. Análisis de un quinquenio. Dermatol Perú 2017;27:74-78.

5. Pereira AR, Porro AM, Seque CA, Pasin VP, et ál. Interconsulta hospitalaria en dermatología en una unidad de trasplante renal. Actas Dermosifiliogr 2018;109:900-907.

6. Falanga V, Schachner LA, Rae V, Ceballos PI, et ál. Dermatologic Consultations in the Hospital Setting. Arch Dermatol 1994;130:1022-1025.

7. Peñate Y, Guillermo N, Melwani P, Martel R, et ál. Dermatologists in Hospital Wards: An 8-Year Study of Dermatology
Consideramos que la participación del especialista puede mejorar la precisión diagnóstica de los pacientes en el contexto hospitalario y agregar un efecto positivo a la atención y la calidad de vida ${ }^{15}$. Aunque pocos pacientes ingresaron directamente por orden del dermatólogo, como observamos en este estudio, un gran porcentaje de ellos presentaban enfermedades cutáneas que requerían tratamiento o hallazgos en la piel que eran importantes para el diagnóstico y el manejo de enfermedades sistémicas. Además, creemos que la instancia de la interconsulta genera un espacio no solo para la comunicación entre los médicos tratantes, sino también para el intercambio de conocimientos, dando lugar al aprendizaje continuo, principalmente para los médicos residentes no dermatólogos ${ }^{7}$.

Creemos que nuestros resultados podrían ser extrapolables a otros hospitales de tercer nivel del territorio nacional que atiendan a una población de características similares a la nuestra.

Consultations. Dermatology 2009;219:225-231.

8. Mancusi S, Festa Neto C. Inpatient dermatological consultations in a university hospital. Clinics 2010;65:851-855.

9. Stanley C, Rubinstein A, Kaler M, Burgos M. Situación y respuesta a las infecciones de transmisión sexual. Boletín epidemiológico sobre el VIH, SIDA e ITS en la Argentina 2018;35:12-18.

10. Özyurt S, Kelekçi K, Şeremet S, Özçelik S. Analysis of Inpatient Dermatologic Consultations. Actas Dermosifiliogr 2014;105:799800.

11. Tay LK, Lee HY, Thirumoorthy T, Pang SM. Dermatology referrals in an East Asian tertiary hospital: a need for inpatient medical Dermatology. Clin Exp Dermatol 2011;36:129-134.

12. Bauer J, Maroon M. Dermatology inpatient consultations: A retrospective study. J Am Acad Dermatol 2010;62:518-519.

13. Fuentes-Suárez A, Domínguez-Soto L. Menosprecio hacia la Dermatología y su repercusión. Rev Med Inst Mex Seguro Soc 2015;53:250-253.

14. Afsar F. Analysis of pediatric dermatology inpatient consultations in a pediatric teaching hospital. Arch Argent Pediatr 2017;115:377-384.

15. Biesbroeck K, Shinohara M. Inpatient consultative dermatology. Med Clin North Am 2015;99:1349-1364. 